\title{
Nanoscale Metal-Metal Contact Physics from Molecular Dynamics: The Strongest Contact Size
}

\author{
Hojin Kim and Alejandro Strachan* \\ School of Materials Engineering and Birck Nanotechnology Center, Purdue University, West Lafayette, Indiana 47907, USA
}

(Received 22 November 2009; published 28 May 2010)

\begin{abstract}
Using molecular dynamics we find that the tensile strength of the contacts between two clean platinum surfaces with nanoscale asperities is strongly size dependent with a maximum strength for contact lengths of approximately $5 \mathrm{~nm}$. This is the first time a strongest size is observed in single crystals. The strengthening with decreasing size down to $5 \mathrm{~nm}$ results from a decrease in the initial density of mobile dislocations available for plastic deformation and the subsequent weakening originates from a reduction in the constraint to mechanical deformation inside the contact by the bulk.
\end{abstract}

DOI: 10.1103/PhysRevLett.104.215504

Processes resulting from the interaction between contacting surfaces including friction and stiction (static friction) are ubiquitous in traditional and emerging technologies including micro- and nanoscale contacting switches for communications [1] and low-power electronics [2]. The performance and reliability of these devices depends on the contact physics that govern the pullout force required to open the switch and on the defect generation that leads to an increase in contact resistance. The interaction between surfaces depends on the materials involved [3], surface chemistry, and roughness as well as operating conditions. In this Letter we focus on clean platinum slabs with nanoscale surface roughness where the interaction is dominated by the metallic bridges that form when surface asperities come into contact. In actual micro- and nanoswitches a large number of nanoscale contacts will form with a size distribution and local forces that depend on surface roughness and chemistry and external closing force [4]. The mechanical response of materials with such nanoscale dimensions is size dependent and the sub-100 $\mathrm{nm}$ range remains vastly unexplored. This factor contributes to the current lack of an understanding in the area of nanoscale contacts. Here we report on a molecular dynamics study of the strength of nanoscale contacts (tensile stress required to open the contact) between clean platinum surfaces as a function of their size. Our simulations shed light into the atomic mechanisms that govern size effects in contact physics and indicate that nanocontact experiments between clean surfaces would be a powerful tool to interrogate mechanical properties at extremely small scales that are difficult to explore by other means. A thin layer of adsorbates is always present in real microelectromechanical systems (MEMS) surfaces and contributes to surface adhesion; our work focuses on the interaction via metallic bridges that form due to large local forces and heating caused by electrical currents.

Size effects in mechanical properties were first characterized by Hall and Petch in polycrystalline materials and by Brenner in metallic whiskers in the 1950s, with the general observation that smaller is stronger. More recently,
PACS numbers: 62.25.-g, 62.20.F-, 62.20.Qp, 81.07.Lk

size effects in strength have been observed in micropillars [5,6], thin films [7], and nanoindentation [8]. In the case of polycrystals, Hall-Petch strengthening exhibits a maximum at a finite size (typically in the tens of nanometers) and further reduction in grain size leads to weakening $[9,10]$. As in the examples above, our molecular dynamics (MD) simulations show that contact strength follows the general trend of smaller is stronger, but only down to approximately $5 \mathrm{~nm}$ where a strongest contact size is observed with further reduction in size leading to weakening.

Each MD simulation involves two platinum (Pt) slabs with (001) or (111) surfaces, thickness of approximately $20 \mathrm{~nm}$ along the $z$ direction and cross sections varying from $10 \times 10$ to $100 \times 100 \mathrm{~nm}^{2}$. Figure 1 shows a snapshot of initial configuration and geometrical details of all simulation cells are given in the supplementary material [11]. Atomic force microscopy (AFM) analysis of MEMS membranes shows relatively flat and smooth surfaces with a slowly varying profile. Thus, our initial configurations are built with sinusoidal surface profiles given by $Z_{\text {initial }}=$ $\min \left[A \sin \left(2 \pi x / \lambda_{x}\right), A \sin \left(2 \pi y / \lambda_{y}\right)\right]$ on both slabs so that peaks in the bottom and top contacting surfaces are perfectly aligned. The asperity height $A$ is kept constant at
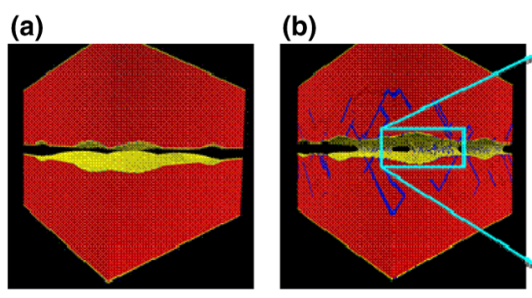

(c)

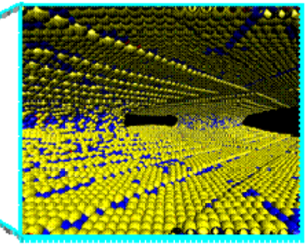

FIG. 1 (color online). Snapshots of the nanoscale metal-metal contact for (001) surfaces with peak-to-peak distance of $25 \mathrm{~nm}$. (a) Initial configuration with fresh four asperities. Light gray (yellow) atoms represent surface and gray (red) atoms are for fcc atoms. (b) Closed Pt-Pt contact. Black (blue) atoms correspond to hcp atoms and denote the motion of partial dislocations.

(c) Close view of an individual metallic nanoscale bridge during contact. 
$1 \mathrm{~nm}$ for all simulations and the peak-to-peak distances in the $x$ and $y$ directions $\left(\lambda_{x}\right.$ and $\left.\lambda_{y}\right)$ are half of the corresponding simulation cell lengths; this leads to four asperities per slab; see Fig. 1(a) and supplementary material [11]. In this way, we control the curvature of the asperities and, consequently, the effective contact area during closing by changing the cross section of the simulation cells. To simulate cyclic closing and opening of the contact we use the following procedure: (i) The two slabs are placed with an initial separation between peaks of $1 \mathrm{~nm}$ and the system is thermalized for $30 \mathrm{ps}$. (ii) To close the contact, external forces are applied to each slab along the $-z$ and $+z$ directions and a constant energy ( $N V E$ ensemble) MD simulation is performed for $180 \mathrm{ps}$. The force is applied to atoms in a thin region $(4.5 \mathrm{~nm})$ at the free surface opposite to the contact surfaces; in all cases the external force is chosen to result in an overall compressive stress (ratio between total force and the cross-sectional area of the simulation cell) of $-100 \mathrm{MPa}$. (iii) In order to open the contact, the applied force is reversed in a stepwise fashion. We use steps of $100 \mathrm{MPa}$ (with resulting stress of 0 , $100 \mathrm{MPa}, 200 \mathrm{MPa}, \ldots)$. For each stress level an $N V E$ simulation is performed for $10 \mathrm{ps}$. Opening the contact requires between 7 and 18 stress increments and the resulting structure is used as the initial configuration for a new contact cycle repeating the entire procedure starting from step (i).

MD simulations were performed using the simulation package LAMMPS [12] with an embedded-atom method (EAM) potential for Pt developed by Baskes and collaborators [13]. For each system we performed between 1 and 25 closing-opening cycles. In order to compute the effective stress at the contacts we calculate the effective contact area by analyzing the atomic coordinates in the thinnest cross section of the contact, see supplementary material for details [11]. To characterize the atomic processes that govern contact physics we analyze the local environment of every atom [11] and classify them into three categories: (i) perfect fcc atoms, (ii) surface atoms, and (iii) atoms with hexagonal close packed (hcp) bonding environments. Planes of hcp atoms play an important role in the plastic deformation of fcc metals: dislocations dissociate into partials that are separated by a stacking fault ribbon (formed by two consecutive rows of hep atoms). Thus, since identifying dislocation lines is challenging in finite temperature, large-scale MD simulations, we will use the number of hcp atoms as a measure of dislocation activity. In configurations consisting of dissociated dislocations the number of hep atoms will be approximately proportional to the total dislocation line length (since the separation between leading and trailing partials is rather constant for a given material). In some nanoscale materials (including various of our contacts) only leading partials are observed nucleating from surfaces or interfaces. In such cases, the number of hcp atoms is also related to the dislocation length but in a more complex, geometry dependent, manner. In these cases the number of hcp atoms is proportional to the area swept by the leading partials. In both these situations the number of hcp atoms provides a useful measure of dislocation content.

As the two surfaces are brought into contact, the asperities form metallic bridges between the two slabs [see Fig. 1]; these bridges need to be broken to open the contact. Figure 2 shows the strength of the metallic bridges (calculated from the force required to open the contact and using the effective contact area computed when the contact is closed) as a function of contact length $\left(L_{c}\right.$, defined as the square root of the effective contact area per asperity) for (001) and (111) surfaces; we show results for fresh surfaces that interact for the first time and for repeated contact. The main results are (i) the stress required to open the nanoscale contacts increases with decreasing contact length down to $L_{c} \sim 5 \mathrm{~nm}$ when a strongest contact size is observed, (ii) further decrease in contact size leads to weaker contacts, and (iii) for the same contact area, fresh contacts are stronger than those that have undergone multiple cycles. Before discussing the atomic origins of these observations we assess the accuracy of our predictions. The inset of Fig. 2 shows the pullout force as a function of contact area from recent AFM experiments on nanoscale $\mathrm{Au}$ contacts [4] together with our MD results including incommensurate (001) surfaces (with a $45^{\circ}$ relative rotation, see [11]). The experimental value is about $60 \%$ smaller than the incommensurate MD result, providing an important validation of our results. Bulk $\mathrm{Pt}$ and $\mathrm{Au}$ have similar tensile strengths, and the free surface energy predicted by the EAM potential we used is similar to the experimental value for $\mathrm{Au}$; thus the strength of nanoscale contacts should be comparable. Our overestimation of the pullout force is likely due to the possible presence of adsorbates and slower opening rates in the experiments.

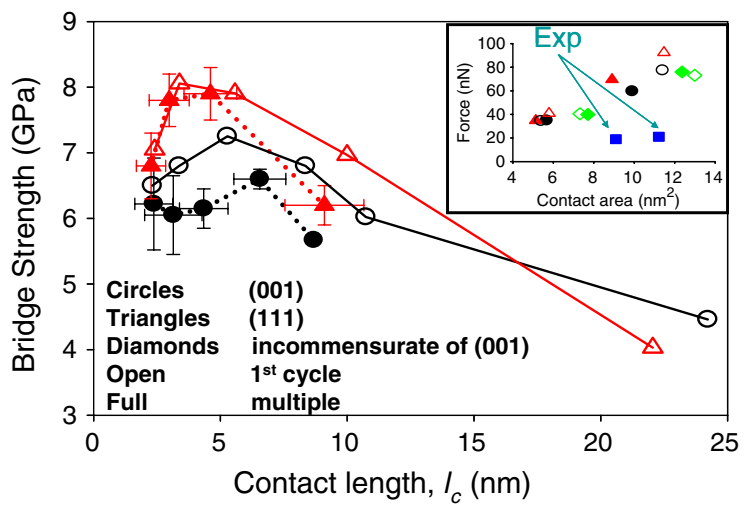

FIG. 2 (color online). Tensile strength of metallic bridge as a function length for (001) and (111) surface; inset compares predicted pullout force with AFM experiment. Open and full symbols represent first cycle with fresh asperities and multiplecycle results, respectively. Error bars denote to the standard deviation for multiple contacts. 
While the fast pullout rates typical of MD simulations are likely to result in increased strength we expect our main conclusions and trends to remain valid for the lower rates of experiments; see Ref. [14]. We performed a simulation at a lower pullout rate $(50 \mathrm{MPa} / 10 \mathrm{ps})$ for a simulation cell with $25 \mathrm{~nm} \times 25 \mathrm{~nm}$ cross-sectional area and observed only a $4 \%$ decrease in strength. Despite these challenges, the direct comparison between MD simulations and AFM experiments for tensile strength of contacts and the level of agreement are unprecedented.

The observed strengthening with decreasing characteristic size for diameters down to approximately $5 \mathrm{~nm}$ is consistent with the generally observed trend of smaller is stronger which results from dislocation-based plasticity becoming increasingly difficult $[6,15]$. Several mechanisms can lead to this behavior: (i) a decrease in the initial density of dislocations available for plasticity, (ii) a decrease in their mobility, or (iii) a difficulty to increase the density of mobile dislocations during deformation [6]. To characterize the underlying physics behind the observed size dependence of contact strength we study how the number of dislocations before opening and the production of new dislocations during deformation depend on contact size, see Fig. 3. Circles denote the number of hcp atoms per unit contact area $\left(\gamma_{\mathrm{hcp}}\right)$ available before opening (defined as the minimum $\gamma_{\mathrm{hcp}}$ after contact closing) for the initial contact and averages over multiple contacts. This is a measure of the dislocations created during contact closing

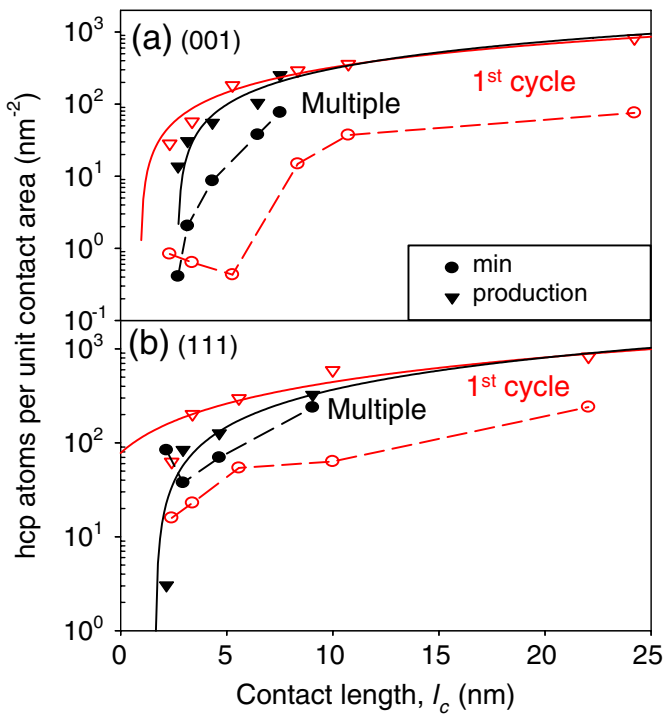

FIG. 3 (color online). Defects available before and produced during contact opening as a function of contact length for (a) (001) and (b) (111) surfaces. Circles and triangles denote the number of hcp atoms per unit contact area available before opening and their production during opening, respectively. Open symbols represent the first cycle with fresh asperities and full ones show multiple-cycle results. Solid lines represent linear fits of the production of dislocations with contact length $L_{c}$ and the dashed lines are to guide the eye. (for the fresh contacts) or accumulated during cyclic operation of the contact; note that defects accumulate during cyclic loading. Triangles in Fig. 3 show the production of hcp atoms during opening (defined as the difference between the maximum $\gamma_{\mathrm{hcp}}$ during opening and the minimum $\gamma_{\text {hcp }}$ after closing). Figure 4 shows snapshots corresponding to the key stages during the first contact cycle for (001) contacts of three different sizes. The snapshots for (111) contacts exhibit the same trend and are included as supplementary material [11]. The strongest contact size for each orientation is shown in the middle column. Our simulations show that both the strengthening with decreasing contact size and the fact that fresh contacts are stronger than those that have experienced cyclic operation are due to a decrease in the dislocations per unit area available before opening. The strongest contact size occurs when the number of mobile dislocations available before opening becomes zero. For (001) we observe few isolated hep atoms and no dislocations, see Fig. 4(e). For (111) surfaces all hcp atoms form stacking faults or twin boundaries parallel to the contact plane [11]; these defects play no role in plasticity during contact opening since the resolved shear stress is zero. As mentioned above, another possible contributing factor to strengthening is the inability to increase the density of dislocations during deformation. However, our data for the production of hcp atoms during the opening indicate that this is not the case for contacts. For all but the smallest contacts, the number of hcp atoms per unit contact area created during pullout is proportional to contact length showing that the volume of the plastic zone is proportional to $L_{c}{ }^{3}$ (see full lines in Fig. 3). This scaling does not indicate an increased difficulty in the production of new dislocations or their motion with decreasing size. Only very small contacts seem unable to produce enough

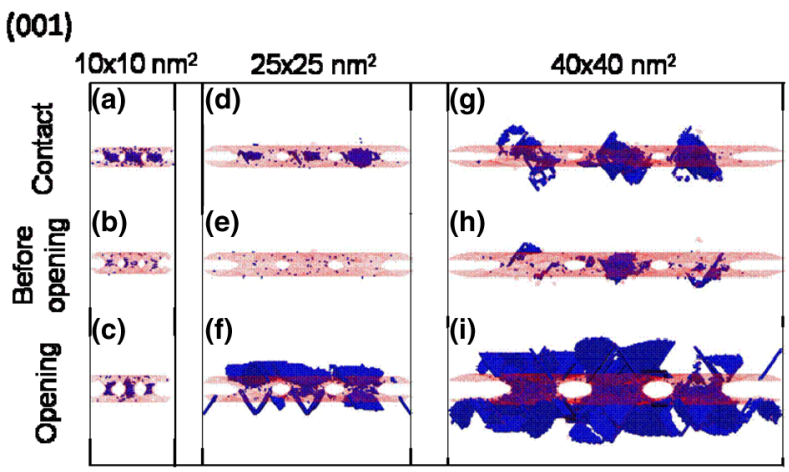

FIG. 4 (color online). Snapshots of the first contact cycle for (001) contacts with different sizes and at various times. The strongest contact size is shown in the middle column. Light (red) and dark (blue) spheres denote surface and hcp atoms, respectively. The top row denotes the configuration after closing; some of the dislocations disappear during the initial stages of opening (middle row) and the number of dislocations increases during opening (the bottom row denotes the configuration with the highest defect density). 
dislocations but these exhibit weakening instead of strengthening. Thus, unlike in nanopillars [6], dislocation starvation does not play an important role in the strengthening of contacts.

Reducing the contact length below $\sim 5 \mathrm{~nm}$ while keeping their height constant leads to a decrease in strength due to reduced constraints to plastic deformation inside the metallic bridges caused by the bulk of the slabs as their aspect ratio $\left(L_{c}\right.$ over contact height $)$ decreases. The available slip planes for dislocation activity make angles of $\sim 54.7^{\circ}$ and $\sim 70.5^{\circ}$ with the tensile axis ( $z$ direction) for (001) and (111) surfaces, respectively; for low aspect ratio bridges with short contact lengths simple glide can occur inside the bridge and plastic deformation is confined inside the contact bridge itself, see Fig. 4(c). For higher aspect ratio cases dislocations must glide into the slabs, see Figs. 4(f) and 4(i), where their motion becomes more difficult due to lower stress and dislocation pileups. This is consistent with the observed decrease in dislocation production observed for the smallest diameters. Furthermore, given the orientation of the active slip planes with respect to the tensile axis, mechanical constraint plays a larger role in (111) surfaces; this explains why the strongest contact size is smaller in (111) surfaces than in (001) ones. This phenomenon has also been proposed as the cause of the observed strengthening of low-aspect ratio microscale tensile tests [16]. Our analysis indicates that the dislocationbased mechanisms proposed here govern the observed size effects in nanoscale contacts. Other phenomena can contribute as well. For example, surface energy and the resulting stress play an important role in the mechanical response of nanoscale materials and its effects on contacts should be further explored.

In summary, our simulations show the strength of nanoscale contacts to be size dependent with a maximum for contact lengths $L_{c} \sim 5 \mathrm{~nm}$ for coherent contacts on (001) and (111); preliminary simulations show similar trends for incommensurate contacts involving (001) surfaces. This is the first time a strongest size is observed in single crystalline metals and it arises from a combination of a decrease in dislocation content with reduced size and constraints to plastic deformation caused by the bulk of the material on the metallic bridge joining the two surfaces. Our results show that single nanoscale contact experiments between clean metallic surfaces of well-characterized curvatures can shed light into the tensile response of materials with size in the sub- $100 \mathrm{~nm}$ regime that are difficult to assess by other techniques. From an application point of view, the irregular roughness of real contacting MEMS surfaces will lead to various contacts with a distribution of sizes, crystal orientations, and local stresses. Since the effective contact area is a small fraction of the apparent contact we expect the interaction between the various contact points to have minimal effects; thus, our results should be useful to describe individual contacts in MEMS switches. We foresee that additional MD simulations using surfaces with realistic roughness, a variety of system sizes, and the presence of adsorbates will help develop a more complete picture of contact physics relevant in microsystems.

This work was supported by the U.S. Department of Energy's National Nuclear Security Administration under contract Grant No. DE-FC52-08NA28617.

*strachan@purdue.edu

[1] G. M. Rebeiz and J. B. Muldavin, IEEE Microw. Mag. 2, 59 (2001).

[2] H. Kam et al., in IEDM Technical Digest: Electron Devices Meeting, 2005 (IEEE International, Washington, DC, 2005), p. 463.

[3] A. Fortini et al., J. Appl. Phys. 104, 074320 (2008).

[4] A. Zong et al., J. Appl. Phys. 100, 104313 (2006).

[5] M. D. Uchic et al., Science 305, 986 (2004).

[6] J.R. Greer and W.D. Nix, Phys. Rev. B 73, 245410 (2006).

[7] Y. Xiang and J. J. Vlassak, Acta Mater. 54, 5449 (2006).

[8] D. K. Ward et al., Proc. Natl. Acad. Sci. U.S.A. 106, 9580 (2009).

[9] S. Yip, Nature Mater. 3, 11 (2004).

[10] J. Schiotz and K. W. Jacobsen, Science 301, 1357 (2003).

[11] See supplementary material at http://link.aps.org/ supplemental/10.1103/PhysRevLett.104.215504 for additional simulation and analysis details.

[12] S. J. Plimpton, J. Comput. Phys. 117, 1 (1995); http:// lammps.sandia.gov.

[13] S. M. Foiles, M. I. Baskes, and M. S. Daw, Phys. Rev. B 33, 7983 (1986).

[14] M. R. Sorensen, M. Brandbyge, and K. W. Jacobsen, Phys. Rev. B 57, 3283 (1998).

[15] V. Yamakov et al., Nature Mater. 3, 43 (2004).

[16] D. Kiener et al., Acta Mater. 56, 580 (2008). 